\title{
Économie politique de la poudre de lait en Afrique de l'Ouest
}

Samuel Pinaud

\section{(2) OpenEdition \\ Journals}

Édition électronique

URL : https://journals.openedition.org/tc/8842

DOI : $10.4000 /$ tc. 8842

ISSN : 1952-420X

Éditeur

Éditions de l'EHESS

\section{Édition imprimée}

Date de publication : 17 mai 2018

Pagination : 30-33

ISBN : 978-2-7132-2750-9

ISSN : 0248-6016

\section{Référence électronique}

Samuel Pinaud, «Économie politique de la poudre de lait en Afrique de l'Ouest », Techniques \& Culture [En ligne], 69 | 2018, mis en ligne le 17 mai 2020, consulté le 29 septembre 2022. URL : http://

journals.openedition.org/tc/8842; DOI : https://doi.org/10.4000/tc.8842 


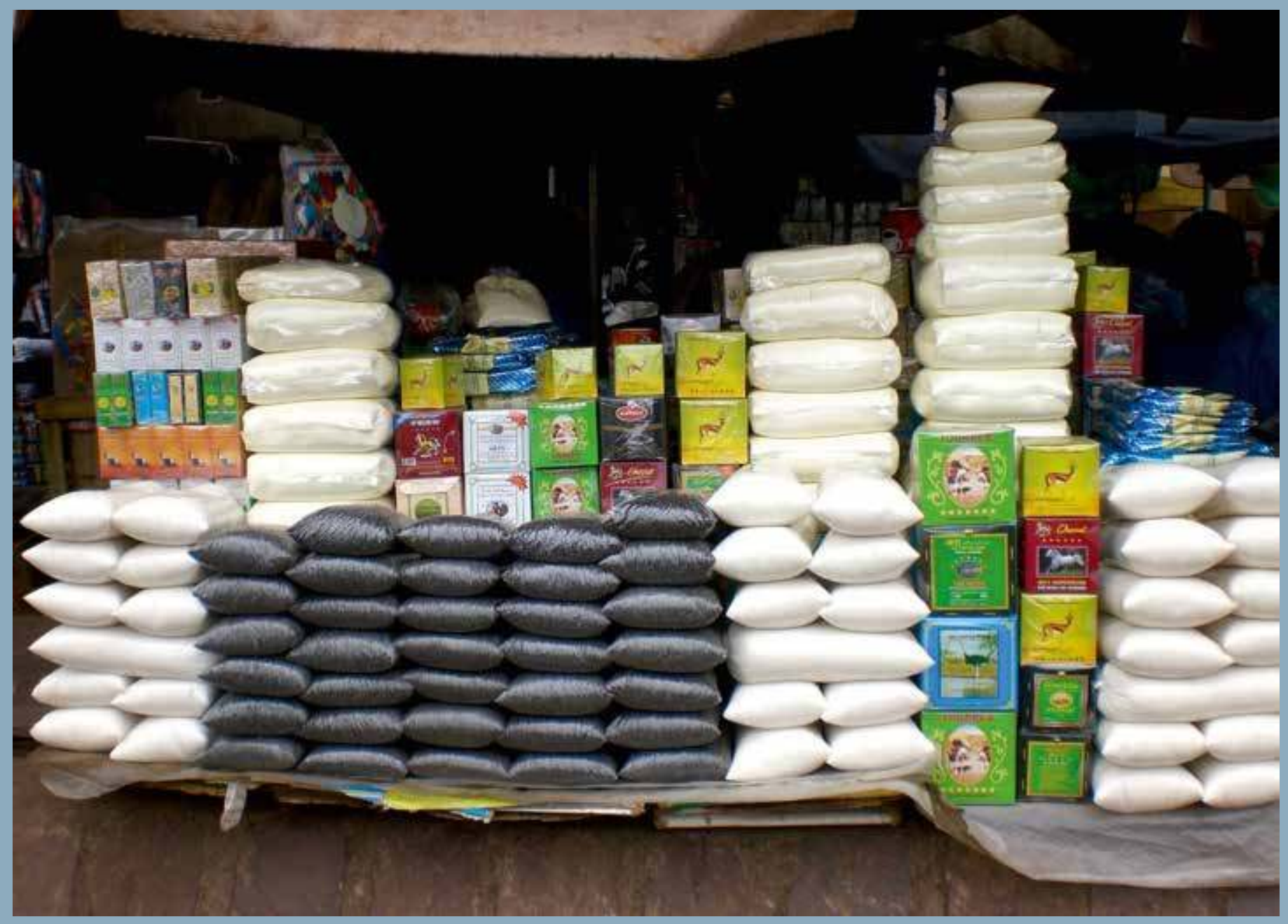




\section{Économie politique de la poudre de lait en Afrique de l'Ouest}

Les techniques industrielles de dessiccation, en enlevant l'eau contenue dans le lait cru, permettent l'obtention d'un «lait de conserve » : la poudre de lait. Additionnée d'eau, la poudre de lait donne un lait liquide aux caractéristiques sanitaires et nutritionnelles proches du lait d'origine.

Cette innovation-produit permet d'entrevoir une distribution de lait désencastrée du système sociotechnique (Akrich 1989) duquel elle était auparavant tributaire: d'abord des techniques de pasteurisation ou de traitement à Ultra haute température (UHT) qui rend le produit aseptique; de la chaîne du froid ensuite qui contrôle le développement microbien. La forte capacité de conservation de la poudre de lait rend techniquement possible une recomposition large de l'économie laitière, tant du point de vue de sa temporalité (stockage possible sur une période relativement longue et dans des conditions sommaires) que de sa géographie (possibilité d'échange à distance à moindre coût) ${ }^{1}$.

Le déploiement de cette économie de la poudre de lait dans un contexte singulier, ici celui d'une grande capitale d'Afrique de l'Ouest (Bamako), donne à voir le rôle des techniques de conservation dans la transformation des échanges alimentaires au sein de la mondialisation contemporaine. Rebut des économies agricoles productivistes privilégiant la consommation de produits laitiers «frais», la poudre de lait est la base de plus de $90 \%$ des volumes de produits laitiers consommés à Bamako (Pinaud 2014). La poudre de lait y est utilisée pour la préparation de mets traditionnels (lait caillé ou déguè2), en accompagnement du café ou du thé, dans le cadre d'un petit-déjeuner de type occidental ou d'une consommation alimentaire de rue caractéristique de l'urbanisation de la vie quotidienne dans la sous-région (allongement de la distance travail/domicile; individualisation des dépenses alimentaires...) (Malvy et al. 1998). Ce produit est aujourd'hui largement consommé -malgré une différenciation forte selon le niveau de revenu - si bien que les consommateurs préfèrent globalement la poudre de lait importée au lait local - disponible en quantité restreinte - non pas tant pour son prix que pour son accessibilité et sa capacité de conservation (Coulibaly et al. 2008) ${ }^{3}$. 
Carrefour des chaises, l'une des entrées du marché central de Bamako dit «marché de Dabanani »

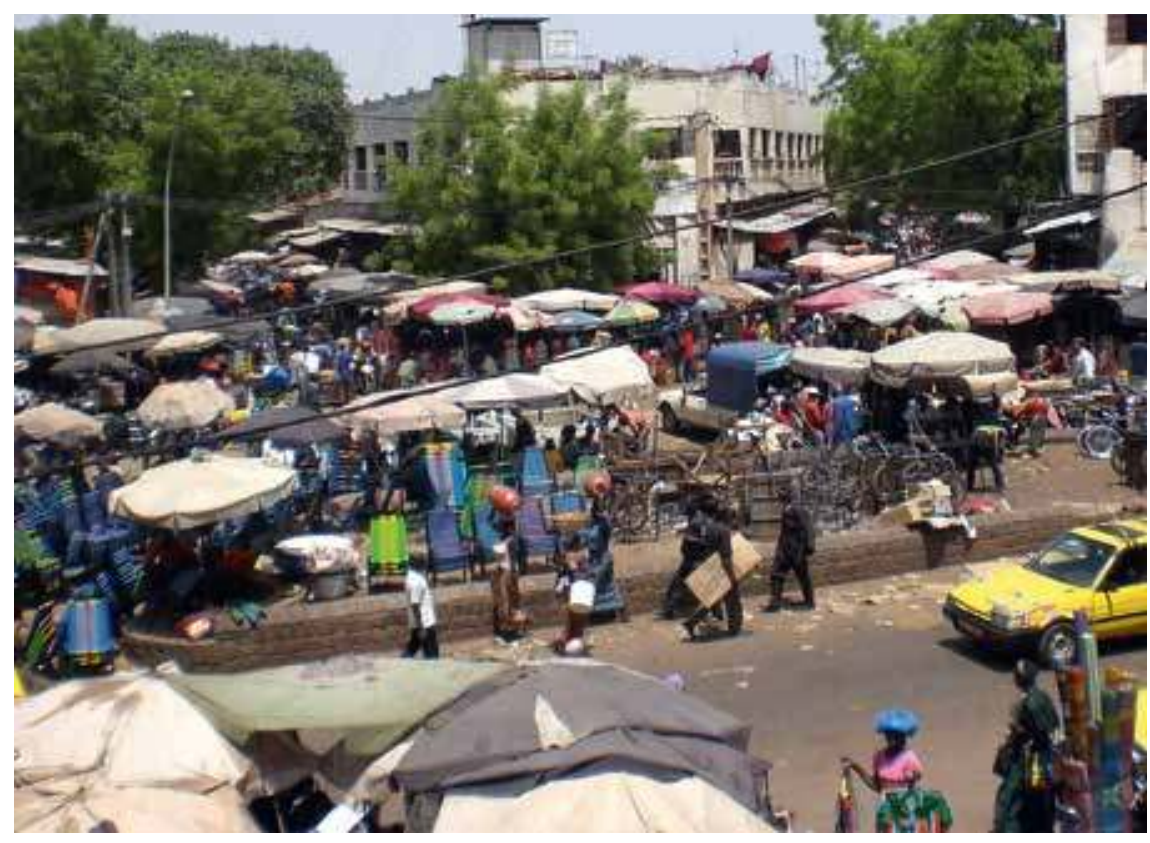

Le pouvoir de conservation de la poudre de lait permet en effet d'«étendre le bras» pour échanger au loin. Toutefois, pour que l'échange ait lieu, le produit doit également être investi d'une certaine valeur sociale. Celle-ci s'est constituée en contexte colonial où la poudre de lait est venue appuyer la reproduction du pouvoir colonial (nourrir les enfants de colons dans des conditions sanitaires similaires à la métropole). La valeur sociale de la poudre de lait s'est renforcée et étendue ensuite à l'ensemble de la population par les politiques humanitaires massives d'aide alimentaire. Cette aide se tarissant avec la baisse des excédents agricoles, les importations marchandes de poudre de lait ont cru, au Mali comme dans l'ensemble de l'Afrique de l'Ouest, de manière exponentielle dans un contexte urbain marqué par une croissance démographique à l'identique, le manque d'infrastructures frigorifiques et d'appui au développement de la production locale, ainsi que des campagnes marketing puissantes d'entreprises multinationales (comme Nestlé).

Le pouvoir de conservation de la poudre de lait, proche d'autres produits en vrac (céréales, café, etc.), permet à sa commercialisation de s'opérer via des canaux commerciaux déjà structurés. Trois formes de valorisation marchande peuvent être distinguées et tiennent à ce pouvoir de conservation particulier. D'abord, en supportant un stockage relativement long sans dégradation de qualité sanitaire ou gustative, la poudre de lait permet aux commerçants de spéculer sur l'évolution de sa valeur marchande. Ensuite, le fait que sa capacité de conservation ne découle ni d'un procédé de conditionnement (comme la conserve) ni d'un ajout de conservateur (sel, sucre, etc.) laisse le commerçant libre de faire varier le conditionnement du produit sans en altérer la qualité pour s'ajuster au pouvoir d'achat (baisse du volume sans variation unitaire du prix) ou dans l'espoir d'un gain (changer le volume sans en avertir le consommateur) comme pour tout autre produit vendu en vrac (céréales, café, etc.). Finalement, les commerçants s'appuient sur le caractère relativement standardisé du produit lié au procédé industriel dont il est issu. Lapparence du produit n'informant qu'imparfaitement sur sa qualité, ils peuvent baisser la qualité de la poudre de lait, soit pour laisser le produit accessible en période de hausse de prix, soit pour augmenter plus directement son profit unitaire, à l'insu du consommateur. 
Les échanges alimentaires ainsi tissés illustrent les transformations contemporaines de la mondialisation. Ils sont marqués par une forte volatilité des prix caractéristique de la financiarisation des marchés agricoles qu'illustrent les stratégies spéculatives des négociants internationaux (Pinaud 2014) et des commerçants bamakois. En outre, ils perdent progressivement leur caractère postcolonial à mesure que l'élevage des pays émergents s'industrialise et que ces derniers deviennent les principaux exportateurs de poudre de lait en Afrique de l'Ouest.

\section{En ligne}

Retrouver l'article complet sur journals.openedition.org/tc: TechniquesECulture 69 «Le temps des aliments».

\section{Notes}

1. Il est évidemment possible de transporter du lait UHT par avion ou bateau, mais les coûts de transport sont importants, notamment en comparaison de la poudre de lait dont un kilogramme permet de reconstituer plus de sept litres de lait liquide. De la même manière, il existe dans plusieurs régions du monde un commerce de citerne de lait frais, mais celui-ci reste contraint, techniquement et donc géographi-

quement, par le caractère périssable du lait transporté sous forme de lait cru réfrigéré (Pinaud 2014).

2. Le déguè est un mélange de lait caillé et de céréales.

3. La production de poudre de lait demande une industrialisation importante de la production laitière, inexistante dans cette région du monde. La poudre de lait disponible est donc toujours importée.

\section{Iconographie}

Image d'ouverture. Un étal de produits alimentaires vendus en vrac. Au premier plan, se trouvent empilés du

sucre, du thé et du café, au second du thé et de la poudre de lait. Crédits des images: (c) Samuel Pinaud, 2009.

\section{I'auteur}

Samuel Pinaud est maître de conférences à l'université Paris-Dauphine, chercheur à l'Irisso. Ses travaux portent sur les transformations contemporaines des marchés et des entreprises agricoles, en France (Hauts-de-France) et en Afrique de l'Ouest (Mali, Burkina Faso).

\section{Références}

Akrich, M. 1989 «La construction d'un système sociotechnique. Esquisse pour une anthropologie des techniques», Anthropologie et société 13: 31-54.

Coulibaly, D. et al. 2008 Recherche sur le mode de gestion du troupeau pour une exploitation économique et durable des bovins laitiers dans les zones périurbaines du Mali. Production, commercialisation, et consommation de lait et produits laitiers en zones périubaines du Mali. Bamako: Rapport de Recherche.
Malvy, D. et al. 1998 «Alimentation de rue, mutations urbaines et différenciations sociales à Bamako (Mali)», Sciences sociales et santé 16: 33-59.

Pinaud, S. 2014 La poudre de lait, le trader parisien et le commerçant bamakois. Une sociologie économique de la mondialisation. Université Paris Ouest Nanterre La Défense: Thèse de doctorat.

\section{Pour citer l'article}

Pinaud, S. 2018 «Économie politique de la poudre de lait en Afrique de l'Ouest», TechniquesE Culture 69 «Le temps des aliments», p. 30-33. 